\title{
HUBBLE TARANTULA TREASURY PROJECT: UNRAVELING TARANTULA'S WEB. I. OBSERVATIONAL OVERVIEW AND FIRST RESULTS*
}

\author{
E. Sabbi ${ }^{1}$, J. Anderson ${ }^{1}$, D. J. Lennon ${ }^{2}$, R. P. van der Marel ${ }^{1}$, A. Aloisi ${ }^{1}$, M. L. Boyer ${ }^{3,4}$, M. CignONi ${ }^{5,6}$, \\ G. de Marchi ${ }^{7}$, S. E. De Mink ${ }^{1,8,18}$, C. J. Evans ${ }^{9}$, J. S. Gallagher III ${ }^{10}$, K. Gordon ${ }^{1}$, D. A. Gouliermis ${ }^{11}$, E. K. Grebel ${ }^{12}$, \\ A. M. Koekemoer ${ }^{1}$, S. S. Larsen ${ }^{13}$, N. Panagia ${ }^{1,14,15}$, J. E. Ryon ${ }^{10}$, L. J. Smith ${ }^{16}$, M. Tosi ${ }^{6}$, And D. Zaritsky ${ }^{17}$ \\ ${ }^{1}$ Space Telescope Science Institute, 3700 San Martin Drive, Baltimore, MD 21218, USA; sabbi@ stsci.edu \\ 2 ESA-European Space Astronomy Center, Apdo. de Correo 78, E-28691 Villanueva de la Cañada, Madrid, Spain \\ ${ }^{3}$ Observational Cosmology Lab, Code 665, NASA, Goddard Space Flight Center, Greenbelt, MD 20771, USA \\ ${ }^{4}$ Oak Ridge Associated Universities (ORAU), Oak Ridge, TN 37831, USA \\ ${ }^{5}$ Dipartimento di Astronomia, Università degli Studi di Bologna, via Ranzani, I-40127 Bologna, Italy \\ ${ }^{6}$ Istituto Nazionale di Astrofisica, Osservatorio Astronomico di Bologna, Via Ranzani 1, I-40127 Bologna, Italy \\ ${ }^{7}$ Space Science Department, European Space Agency, Keplerlaan 1, 2200-AG Noordwijk, The Netherlands \\ 8 Johns Hopkins University, 3400 North Charles Street, Baltimore, MD 21218, USA \\ ${ }^{9}$ UK Astronomy Technology Center, Royal Observatory Edinburgh, Blackford Hill, Edinburgh EH9 3HJ, UK \\ ${ }^{10}$ Department of Astronomy, University of Wisconsin, 475 North Charter Street, Madison, WI 53706, USA \\ ${ }^{11}$ Zentrum für Astronomie, Institut für Theoretische Astrophysik, Universität Heidelberg, Albert-Ueberle-Str. 2, D-69120 Heidelberg, Germany \\ ${ }^{12}$ Zentrum für Astronomie, Astronomisches Rechen-Institut, Universität Heidelberg, Mönchhofstr. 12-14, D-69120 Heidelberg, Germany \\ ${ }^{13}$ Department of Astrophysics/IMAPP, Radboud University Nijmegen, P.O. Box 9010, 6500-GL Nijmegen, The Netherlands \\ ${ }^{14}$ Istituto Nazionale di Astrofisica, Osservatorio Astrofisico di Catania, Via Santa Sofia 78, I-95123 Catania, Italy \\ ${ }^{15}$ Supernova Limited, OYV 131, Northsound Road, Virgin Gorda, British Virgin Islands \\ ${ }^{16}$ ESA/STScI, 3700 San Martin Drive, Baltimore, MD 21218, USA \\ ${ }^{17}$ Steward Observatory, University of Arizona, 933 North Cherry Avenue, Tucson, AZ 85721, USA \\ Received 2013 April 22; accepted 2013 July 1; published 2013 July 30
}

\begin{abstract}
The Hubble Tarantula Treasury Project (HTTP) is an ongoing panchromatic imaging survey of stellar populations in the Tarantula Nebula in the Large Magellanic Cloud that reaches into the sub-solar mass regime $\left(<0.5 M_{\odot}\right)$. HTTP utilizes the capability of the Hubble Space Telescope to operate the Advanced Camera for Surveys and the Wide Field Camera 3 in parallel to study this remarkable region in the near-ultraviolet, optical, and near-infrared spectral regions, including narrow-band $\mathrm{H} \alpha$ images. The combination of all these bands provides a unique multi-band view. The resulting maps of the stellar content of the Tarantula Nebula within its main body provide the basis for investigations of star formation in an environment resembling the extreme conditions found in starburst galaxies and in the early universe. Access to detailed properties of individual stars allows us to begin to reconstruct the temporal and spatial evolution of the stellar skeleton of the Tarantula Nebula over space and time on a sub-parsec scale. In this first paper we describe the observing strategy, the photometric techniques, and the upcoming data products from this survey and present preliminary results obtained from the analysis of the initial set of near-infrared observations.
\end{abstract}

Key words: galaxies: star clusters: individual (30 Doradus) - Magellanic Clouds - stars: formation - stars: imaging - stars: pre-main sequence

Online-only material: color figures

\section{INTRODUCTION}

The Tarantula Nebula (a.k.a. 30 Doradus, hereafter "30 Dor") in the Large Magellanic Cloud (LMC) is one of the most famous objects in astronomy. The first astronomical references to the Tarantula Nebula are more than 150 years old (de la Caille 1761; Herschel 1847). For more than a decade the Tarantula's ionizing cluster, Radcliffe 136 (R136), was thought to be the most massive known single star (250-1000 $M_{\odot}$; Feitzinger et al. 1980). Apart from a few dissonant voices (e.g., Feast 1953; Walborn 1973; Moffat 1982), the true nature of R136 continued to elude the majority of the astronomical community until Weigelt \& Baier (1985) resolved it into eight components by using holographic speckle interferometry.

The Tarantula Nebula and R136 are now considered one of the few known starbursts in the Local Group. The size of the Tarantula Nebula ( $200 \mathrm{pc}$ in diameter) and "local" density

\footnotetext{
* Based on observations with the NASA/ESA Hubble Space Telescope, obtained at the Space Telescope Science Institute, which is operated by AURA Inc., under NASA contract NAS 5-26555.

18 Hubble Fellow.
}

of OB stars parallel those observed in systems characterized by very intense star formation, such as the starburst knots observed in interacting galaxies in the local universe and young galaxies at high redshift $(z>5$; Meurer et al. 1997; Shapley et al. 2003; Heckman et al. 2004). In a recent paper Selman \& Melnick (2013) inferred a total stellar density in the core of R136 between $\rho_{0} \geqslant 1.5 \times 10^{4} M_{\odot} \mathrm{pc}^{-3}$ and $10^{7} M_{\odot} \mathrm{pc}^{-3}$. Thus dissecting the stellar populations and inferring an accurate description of the anatomy of the Tarantula Nebula allows us to reconstruct for the first time the temporal and spatial evolution of a prototypical starburst on a sub-cluster scale.

In this paper we present an introductory overview of the "Hubble Tarantula Treasury Project" (HTTP, PI: Sabbi, GO-12939), an ongoing treasury program designed to observe the entire region with the Hubble Space Telescope (HST) in the near-ultraviolet (NUV, F275W and F336W), optical (F555W, F658N), and near-infrared (NIR, F110W and F160W). The program is built on an existing HST monochromatic survey in the F775W filter (GO-12499, PI: Lennon), designed to measure proper motions of runaway candidates. 
Bias-subtracted and flat-fielded images, as well as all the raw data, are immediately available to the public and can be downloaded from the Mikulski Archive for Space Telescopes (MAST). Photometric catalogs and mosaicked images for all the filters will be distributed by our team to the community in a timely fashion. The survey progress can be followed through the Web site http://30dor.stsci.edu.

This paper is organized as follows: we present the motivations for the survey in Section 2, and the observing strategy in Section 3. As of the writing of this paper, we have collected half of the data in the NIR. The data reduction and the analysis of this portion of the dataset are discussed in Section 4, while the color-magnitude and color-color diagrams (CMDs and CCDs, respectively) are presented in Section 5. A discussion of the results and conclusions can be found in Section 6 .

\section{TRACING THE EVOLUTION OF A STARBURST}

\subsection{The Cosmological Context}

Starbursts are short-lived periods of intense, massive star formation (Searle et al. 1973) that occur in compact regions (10-1000 pc) and dominate the overall luminosity of their host galaxy (Heckman 2005). In their annual review Kennicutt \& Evans (2012) remind us that the star formation rate per unit area in a starburst is much larger than in disk-averaged star formation rate surface densities $\left(\sim 0.1 M_{\odot} \mathrm{yr}^{-1} \mathrm{kpc}^{-2}\right)$. Evidence for recent starburst activity is found in $6 \%$ of the galaxies in the local universe $(z \ll 1$; Lee et al. 2007, 2009) and in $15 \%$ of those at high redshift ( $z>1$; O'Connell 2005; Douglas et al. 2010), indicating that starbursts are a relatively common phenomenon. The impact of a major starburst on the evolution of a galaxy is dramatic, because it will shape the galaxy dynamics, stellar evolution and chemical compositions in ways that are largely dictated by the intensity and the duration of the bursting event (i.e., Dekel \& Silk 1986; Mac Low \& Ferrara 1999; Kennicutt \& Evans 2012).

Starbursts are studied on two complementary fronts. High-redshift surveys collect information over a range of long look-back times for a large sample of objects, but suffer from limited or no spatial information and crude age diagnostics. Studies in the local universe, on the other hand, are affected by the small size of the sample, but can probe discrete structures within the star-forming region using more precise population diagnostics (Hunter \& Elmegreen 2006; Tolstoy et al. 2009; McQuinn et al. 2010). In both approaches the characterization of starburst properties is still rudimentary and sometimes contradictory.

Studies in individual nearby dwarf galaxies and in surveys at redshift higher than $\sim 1.0$ indicate that a starburst may last for 5-10 Myr, suggesting that the violent feedback from the massive stars formed during the burst suppresses for a while any further star formation (Tosi et al. 1989; Ferguson \& Babul 1998; Annibali et al. 2003, 2009; Stinson et al. 2007). Other studies in the local universe, however, advocate for much longer star-forming episodes (>100 Myr; Konstantopoulos et al. 2009; McQuinn et al. 2012), although it is not clear if their approach allows for sufficiently high temporal resolution. Since the spectral energy distributions (SEDs) of galaxies at high redshift can be interpreted only by assuming a duration for the burst, understanding how and for how long star formation can be sustained during a starburst event has profound implications on our picture for understanding galaxies.

\subsection{The Tarantula Nebula: A Unique \\ Opportunity to Decipher Starbursts}

The Tarantula Nebula is by far the most luminous ( $f \mathrm{H} \alpha \sim$ $\left.1.3 \times 10^{-8} \mathrm{erg} \mathrm{cm}^{-2} \mathrm{~s}^{-1}\right)$ and massive star-forming region in the Local Group (Kennicutt \& Hodge 1986). Covering an area of $\sim 40,000 \mathrm{pc}^{2}$, the Tarantula Nebula is the closest extragalactic giant H II region and is comparable in size to the unresolved luminous H II complexes observed in distant galaxies (Oey et al. 2003; Hunt \& Hirashita 2009).

With more than 800 spectroscopically confirmed OB stars (Evans et al. 2011), some of which are among the most massive candidates (Crowther et al. 2010), 30 Dor is often described as a mini-starburst (Leitherer 1998). The UV flux coming from 30 Dor is such that it has been proposed as a small scale local analog to the Lyman-break galaxies (Meurer et al. 1997; Shapley et al. 2003; Heckman et al. 2004). Furthermore several authors (e.g., O'Connell \& Mangano 1978; O'Connell et al. 1995; Brandl et al. 2004) have suggested that the luminous knots observed in bursting galaxies such as M82 are made of (multiple) 30-Dor-like systems.

By virtue of its location in the LMC ( $\sim 50 \mathrm{kpc}$; Panagia et al. 1991; Pietrzyński et al. 2013), with Hubble we can resolve the Tarantula Nebula into single stars down to the sub-solar mass regime (Andersen et al. 2009; De Marchi et al. 2011). The low inclination angle $\left(\sim 30^{\circ}\right.$; Nikolaev et al. 2004) limits the line of sight confusion, and the foreground reddening is low because of the high Galactic latitude.

The Tarantula Nebula is a very dynamic region both in terms of runaway-star candidates (e.g., Evans et al. 2011) and gas motion (Chu \& Kennicutt 1994). Since it is a multistage star-forming region (Walborn \& Blades 1997), where loose associations and very dense star clusters of different ages coexist in a relatively small volume, the Tarantula Nebula is a noteworthy window in which to test various scenarios of clustered star formation. In particular, observational evidence shows that over the last $\gtrsim 25 \mathrm{Myr}$ the star-formation history of the region (Figure 1) has been complex:

1. Walborn \& Blades (1997) identified multiple generations of stars ranging from $\leqslant 0.5 \mathrm{Myr}$ to $\sim 25 \mathrm{Myr}$ in $1^{\prime}-2^{\prime}$ from the core of 30 Dor, R136.

2. Grebel \& Chu (2000) derived an age of 20-25 Myr for Hodge 301 , a cluster $\sim 3^{\prime}$ to the northwest of R136.

3. NICMOS observations at the "frontier" between Hodge 301 and R136 showed that this region is strewn with several massive $\mathrm{O}$ stars embedded in dense knots of dust, suggesting that stellar feedback from the two clusters may be triggering new episodes of star formation (Brandner et al. 2001).

4. $\sim 7^{\prime}$ to the west of R136 the super-bubble created by the $\sim 10 \mathrm{Myr}$ old OB association LH99 is filled by the expanding supernova remnant N157B, which contains the most energetic pulsar known (Chen et al. 2006).

5. Deep HST optical and NIR observations suggest that the core of 30 Dor, R136, is an interacting double cluster (Sabbi et al. 2012).

As these previous studies suggest, the Tarantula Nebula region is complicated. While there have been attempts to study in detail some of its specific parts, much of the focus with HST to date has been on its central cluster (Hunter et al. 1995, 1996; Walborn et al. 1999, 2002; Selman et al. 1999; Brandner et al. 2001; Andersen et al. 2009; De Marchi et al. 2011; Sabbi et al. 2012; Selman \& Melnick 2013). These studies have yielded 


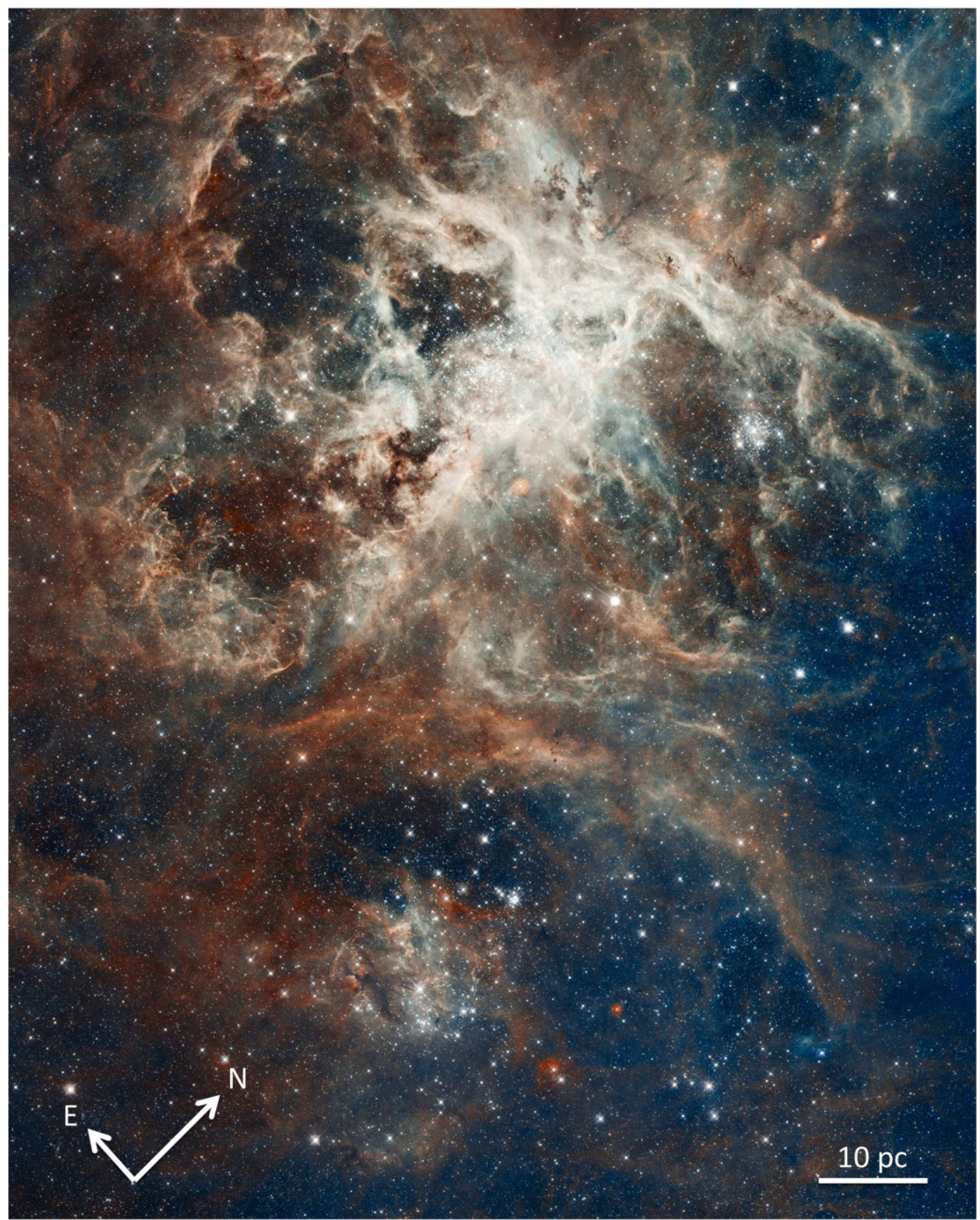

Figure 1. Tarantula Nebula as seen with HST (Credit NASA, ESA, Lennon; GO-12499). The image is the result of 15 HST pointings with the F775W filter, using WFC3 and ACS in parallel. The projected area in the sky is $\sim 14^{\prime} \times 12^{\prime}$. The dynamic range of the image has been compressed to show both bright stars and faint filaments of gas. Colors are from ESO WFI exposures in the $\mathrm{H} \alpha$ (in red) and S II (in blue) filters. The ionizing cluster R136 is located in the middle of the upper half of the image, in the brightest (white) part of the nebulosity. The small bright cluster to the right of R136 is Hodge 301, and the loose stellar association in the middle of the lower half of the image is NGC 2060. Once observations are completed, HTTP will cover approximately the same region in six additional filters, namely, F275W, F336W, F555W, F658N, F110W and F160W.

(A color version of this figure is available in the online journal.)

significant results, nevertheless a comprehensive understanding of the Tarantula Nebula in its entirety can only be reached by detailed studies that include other star clusters and surrounding field stellar populations.

\subsection{The HTTP Science Drivers}

The ongoing HST panchromatic survey of the Tarantula Nebula HTTP (PI: Sabbi, GO-12939) is built on an existing HST program (PI: Lennon, GO-12499) in filter F775W
( $\sim$ Sloan Digital Sky Survey $i$-band). HTTP was awarded 60 orbits of HST time. Figure 2 shows the wavelength coverage of the survey, once it is combined with the GO-12499 dataset.

The decision to survey the region over such a wide wavelength baseline was driven by several factors. Adding an optical filter to the $\mathrm{F} 775 \mathrm{~W}$ dataset allows us to derive CMDs down to $m_{\mathrm{F} 775 \mathrm{~W}}=27$, that for at the distance of the LMC, for a $\sim 2 \mathrm{Myr}$ old stellar population with $Z=0.008$ corresponds to $\sim 0.5 M_{\odot}$. Our photometry goes 4-5 mag below the oldest turn-off and therefore allows us to reconstruct the star formation history 


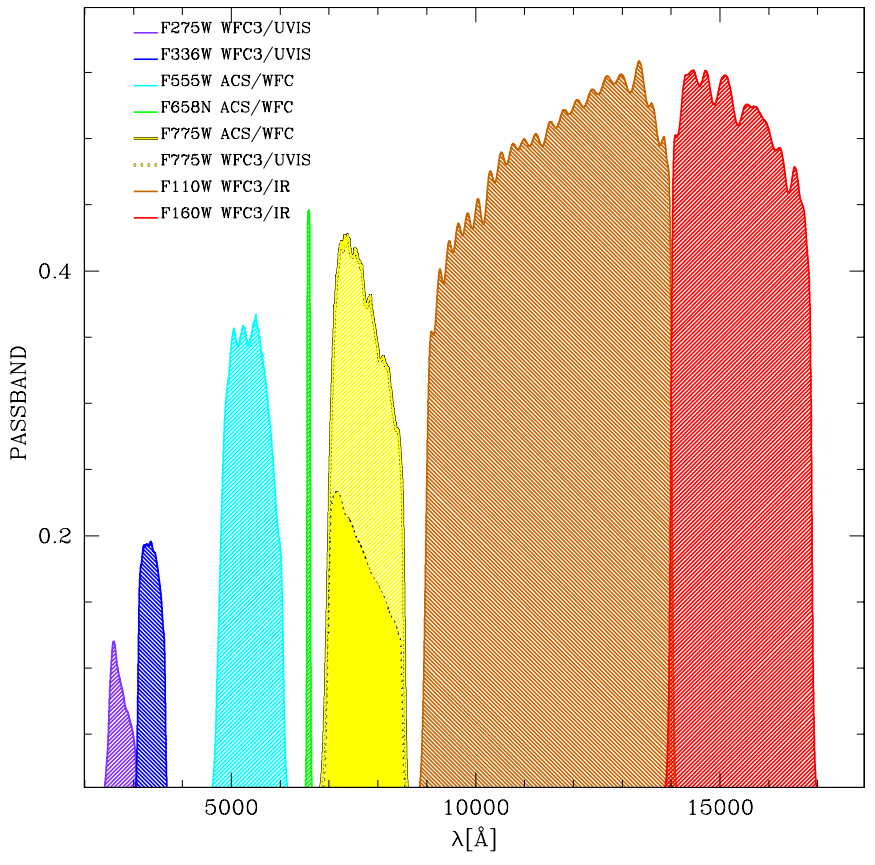

Figure 2. Passband of the filters used in HTTP as a function of wavelength. (A color version of this figure is available in the online journal.)

of the entire region and infer the changes in star formation rate with time. We preferred the Advanced Camera for Surveys (ACS) F555W to the wider F606W filter, because the latter, being quite red, would have provided a limited color baseline relative to the F775W filter. Furthermore the $\mathrm{H} \alpha$ line falls in the F606W bandpass and in $\mathrm{H}$ II regions this can affect the accuracy of the photometry.

Supplementing our dataset with two NIR filters allows us to extend our study to the dustiest regions, in order to better constrain the properties of cool stars such as red supergiant, red giant (RGB), and asymptotic giant branch stars. Even more important, only NIR observations can identify regions of ongoing star formation that are still embedded in their dusty cocoons (see Figure 3).

Previous NICMOS observations have shown that star formation took place in the recent past at the gaseous interface between R136 and Hodge 301 (Brandner et al. 2001). By extending this study to the entire Tarantula Nebula we can identify pockets of ongoing star formation far from the main clusters. Thus we will test the predicted constructive properties of stellar feedback (e.g., Elmegreen \& Lada 1977; Vanhala \& Cameron 1998), and evaluate the efficiency of triggered star formation as inferred from modeling (e.g., Dale et al. 2012). We have selected the F110W and F160W filters since they provide the highest throughput with the IR channel.

Observations in the NUV have been designed to infer the temperature of the hotter stars and, once combined with optical measurements, constrain the amount of extinction (Zaritsky 1999; Romaniello et al. 2002). We have chosen the Wide Field Camera 3 (WFC3) filter F336W because of its high throughput and because it is specifically designed to constrain the Balmer break. To better determine the temperature of the hotter stars and to provide the largest baseline possible to characterize the extinction law we added the even bluer filter F275W.

Finally we are observing the Tarantula Nebula with the narrow-band ACS filter F658N (corresponding to $\mathrm{H} \alpha$ ). Young $(<5 \mathrm{Myr})$ low-mass pre-main sequence (PMS) stars can be easily identified in the optical CMD as a population of faint red objects, and can be used to trace the extent of young star-forming regions (Sabbi et al. 2007, 2012; Cignoni et al. 2010; Gouliermis et al. 2012). With the aging of a stellar population, however, the colors of the PMS stars become too blue to be distinguished from the low-mass MS stars of the field, an effect that becomes even more severe when extinction is highly variable, as is the case for the Tarantula Nebula. In previous studies (Panagia et al. 2000; De Marchi et al. 2010, 2011) we have shown that a combination of broad- and narrow-band filters can be used to identify low-mass PMS stars with active mass accretion, and that this approach is very efficient in picking up older (>10 Myr) and more diffuse episodes of star formation. In addition, the same strategy will allow us to identify main-sequence and evolved stars with $\mathrm{H} \alpha$ excess, such as Be and $\mathrm{B}[\mathrm{e}]$ stars (Grebel 1997).

In summary the high sensitivity, spatial resolution, and broad wavelength coverage of HTTP will allow us to reconstruct the Tarantula's star-formation history in space and time on a parsec scale. In particular we will be able to (1) characterize when and where star formation occurred, (2) establish the length and the strength of the star-forming episodes and their spatial scale, (3) depict the life cycle of star clusters, (4) portray the role of stellar feedback in shaping the evolution of a starburst, and (5) probe the universality of the stellar initial mass function. In addition, a Bayesian fitting of the stellar SEDs will allow us to accurately determine $R_{V}$, and therefore supply information about the size of the dust grains and their spatial distribution (K. Gordon et al. 2013 , in preparation).

\section{DESCRIPTION OF THE OBSERVATIONS}

In both datasets described in this paper (GO-12499, PI: Lennon; and GO-12939, PI: Sabbi) the Wide Field Channel (WFC) of ACS is used in parallel with either the UVIS or the IR channels of WFC3 to maximize the efficiency of the observations. Since there are no filters in common between the WFC3/IR channel and the WFC3/UVIS or ACS/WFC, in this paper we will simply refer to the instrument (ACS and WFC3), without specifying the channel.

\subsection{The GO-12499 Dataset}

The HST program GO-12499 (PI: Lennon) represents the first epoch of a proper-motion survey of massive stars in the 30 Dor region. The survey covers a projected area of $\sim 14^{\prime} \times 12^{\prime}$, corresponding to $\sim 210 \times 180 \mathrm{pc}$ at the distance of the LMC.

A total of 15 pointings were used to map the whole region. All the observations were taken between 2011 October 3 and 29 , using WFC3 as primary instrument and ACS in parallel. The orientation angle of the mosaic was chosen to include the very massive runaway VFTS\#16 (Evans et al. 2010).

Since the goal of the program is to determine the positions of the stars with exquisite accuracy, all the observations were acquired in a single filter. Filter F775W was chosen in an effort to minimize the effect of the strong and variable nebular emission that pervades the entire region.

Each pointing was observed for one orbit of HST time, and each orbit consisted of one short and four deeper exposures with each camera. The exposure times were dictated by the buffer dumping of WFC3 and by the length of the orbit. To give the survey as much spatial uniformity as possible, observations were stepped in such a way that no star would fall in the inter-chip gap in more than one of the WFC3 exposures. 


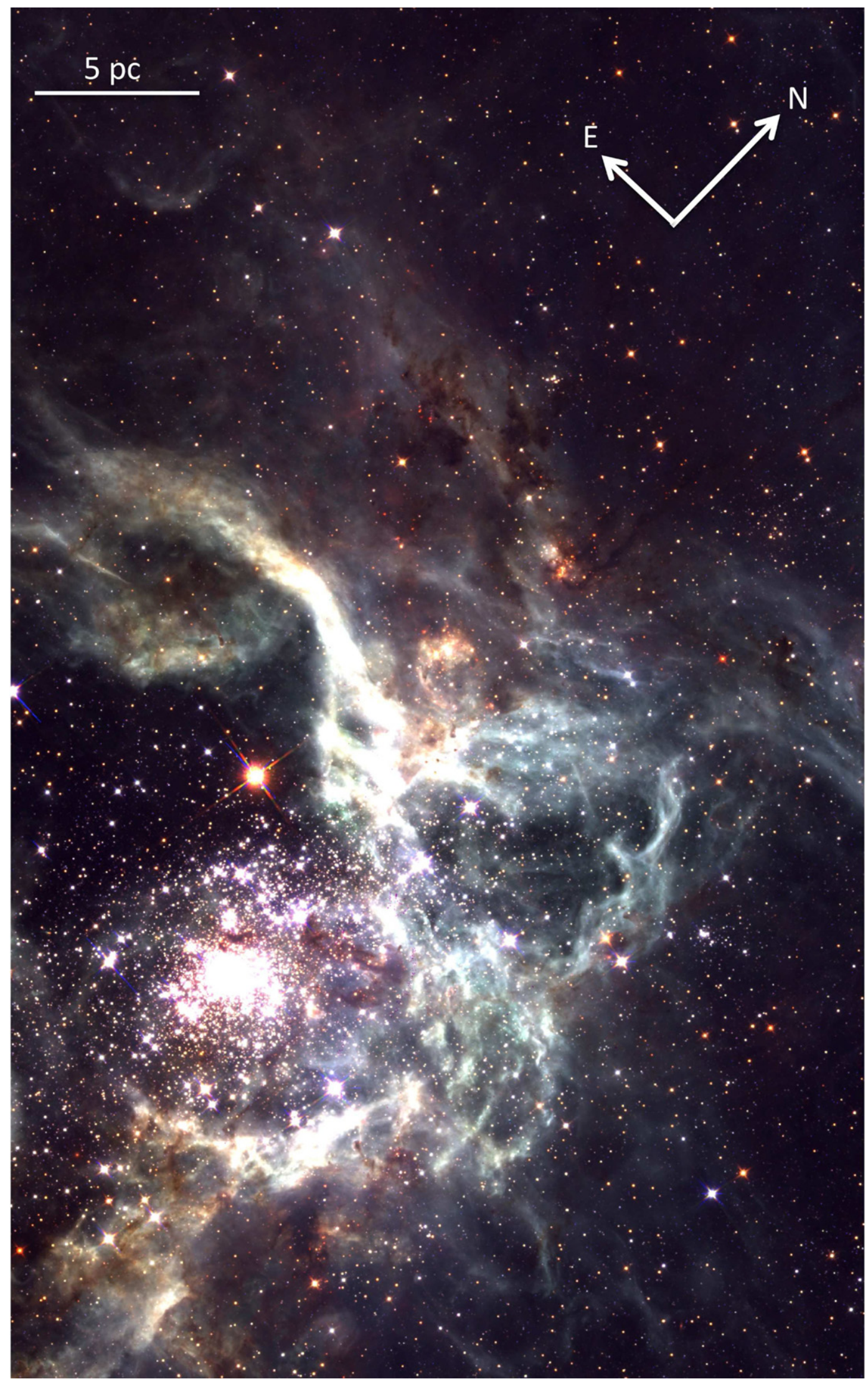

Figure 3. Detail of the HST color-composite image of the Tarantula Nebula, centered on R136. F775W is in blue, F110W is in green, and F160W is in red. The projected area in the sky is $\sim 2^{\prime} 50^{\prime \prime} \times 4^{\prime} 40^{\prime \prime}$.

(A color version of this figure is available in the online journal.) 


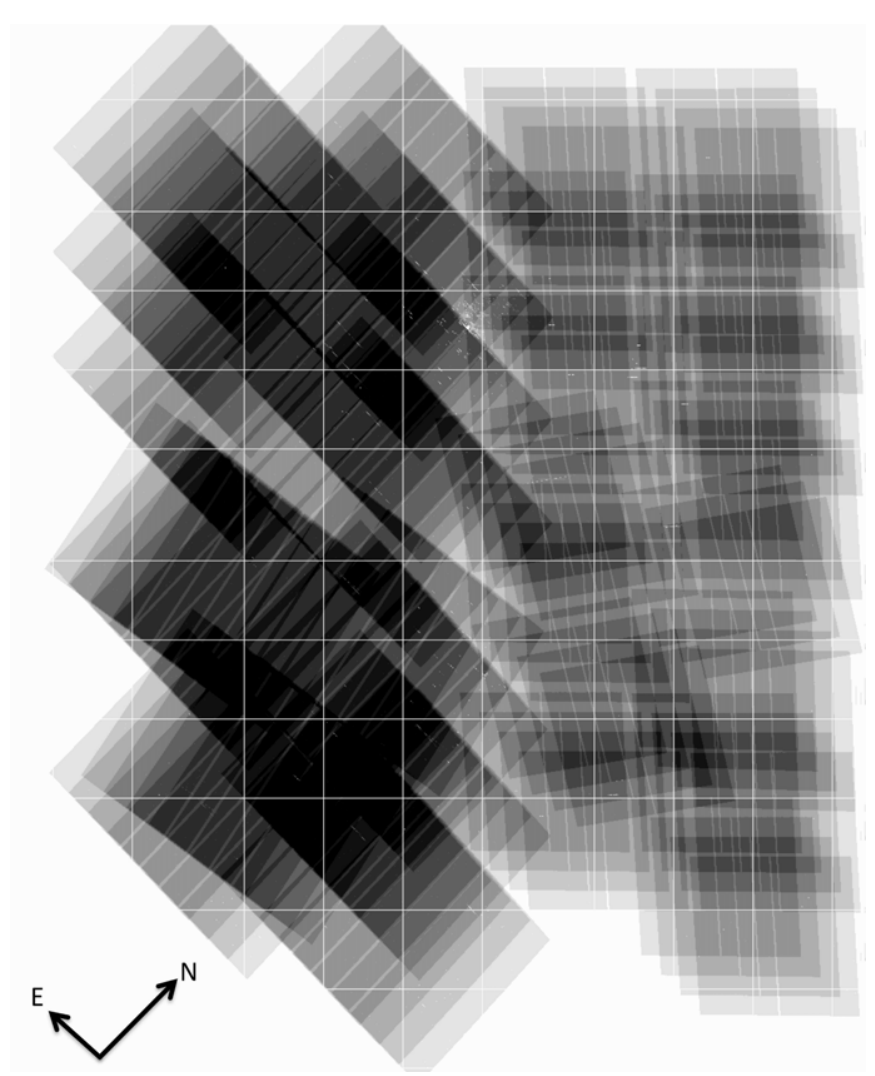

Figure 4. Map of the depth of the mosaic obtained by stacking all the deep exposures of the GO-12499 dataset. The darker the image, the larger the number of overlapping images. WFC3 is on the right and ACS is on the left side of the image.

The full dataset consists of 15 exposures of $35 \mathrm{~s}, 14$ of $500 \mathrm{~s}$, and 45 of $700 \mathrm{~s}$ with UVIS and 15 of $32 \mathrm{~s}, 14$ of $377 \mathrm{~s}$, and 45 of $640 \mathrm{~s}$ with ACS for a total of 148 exposures. Once stacked together the deep exposures result in a mosaic of $\sim 28,000 \times 22,000$ pixels, with a 40.00 mas pixel $^{-1}$ scale. Figure 1 shows the mosaic, with colors added based on ground based data. Figure 4 shows the depth map of the deep stacked image.

The CCD detectors on board HST are subject to a flux of energetic particles that progressively damage the silicon lattice of the detectors and create charge traps that redistribute the flux from one pixel to the other during the detector readout process. As a result of this cumulative radiation damage, the charge transfer efficiency (CTE) of the CCDs on board HST is progressively degrading. We used the algorithm described by Anderson \& Bedin (2010) to correct the effects of the degrading CTE directly on the images. This algorithm is automatically applied by the ACS calibration pipeline. The routine we used to correct WFC3 data for CTE can be downloaded from the WFC3 Web site. ${ }^{19}$

\subsection{The GO-12939 Dataset}

As was done for program GO-12499, we are observing the field with WFC3 as primary instrument and ACS in parallel. Visits are organized in four blocks of visits. Figure 5 shows the 36 NIR and 24 UVIS WFC3 pointings superimposed on the DSS image of 30 Dor, together with the ACS parallels. Each

19 http://www.stsci.edu/hst/wfc3/tools/cte_tools
Table 1

Journal of Observations

\begin{tabular}{lccrc}
\hline \hline Instrument & Filter Name & Number of Exposures & Exposure Time & Post-flash \\
\hline WFC3/UVIS & F275W & 1 & 467 & Yes \\
WFC3/UVIS & F275W & 1 & 697 & Yes \\
WFC3/UVIS & F336W & 1 & 14 & Yes \\
WFC3/UVIS & F336W & 2 & 700 & Yes \\
ACS/WFC & F555W & 1 & 13 & No \\
ACS/WFC & F555W & 1 & 337 & No \\
ACS/WFC & F555W & 3 & 640 & No \\
ACS/WFC & F658N & 1 & 300 & No \\
ACS/WFC & F658N & 3 & 640 & No \\
ACS/WFC & F775W & 1 & 32 & No \\
ACS/WFC & F775W & 1 & 377 & No \\
ACS/WFC & F775W & 4 & 640 & No \\
WFC3/UVIS & F775W & 1 & 35 & No \\
WFC3/UVIS & F775W & 1 & 507 & No \\
WFC3/UVIS & F775W & 4 & 690 & No \\
WFC3/IR & F110W & 1 & 300 & $\ldots$ \\
WFC3/IR & F110W & 1 & 640 & $\ldots$ \\
WFC3/IR & F160W & 2 & 640 & $\ldots$ \\
\hline
\end{tabular}

pointing corresponds to a single orbit of $H S T$ time and consists of a four-step dither-pattern.

The first and third blocks are observed with WFC3/IR and ACS. During each orbit we acquire two exposures in the WFC3 F110W and two in the F160W filters, while ACS is used to collect four observations in the F658N filter (in Figure 5, upper panels, WFC3 is in green and ACS is in magenta).

In the second and fourth blocks, each orbit begins with the acquisition of a short exposure in the F336W filter with WFC3 and a short ACS image in the F555W filter. We then collect two images in the F336W and two in the F275W filters. At the same time, we acquire four exposures in the F555W filter with ACS (in Figure 5, lower panels, WFC3 is in blue and ACS is in magenta).

Half of the IR observations were acquired between 2012 December 12 and 19 and are described in the next section. The remaining half is scheduled for the summer of 2013. The NUV observations will be collected in 2013 April and September. This observing strategy is designed to exploit the natural rotation of the telescope during the year.

The filters, the number of exposures, and the exposure times used in HTTP are summarized in Table 1. Updates on the status of the survey can be found at http://30dor.stsci.edu.

Observations with very low background $\left(<10 e^{-}\right.$for WFC3 and $<50 e^{-}$for ACS (for a discussion of the background levels in UVIS observations, see Baggett \& Anderson 2012), such as those acquired in the NUV and/or through narrow-band filters) suffer large losses for very faint sources, to the point that faint sources can completely vanish during the readout transfers. Since Cycle 20, it has been possible to mitigate the effects of the degrading CTE by adding an extra amount of photons through an internal illumination. This process, called "post-flash," has the effect of artificially increasing the background level (MacKenty \& Smith 2012). All the NUV observations of HTTP are acquired using "post-flash." We decided to not use post-flash for the $\mathrm{H} \alpha$ exposures, since we estimated that the background would be sufficiently high. Even with the background increased, all sources still suffer some CTE loss, and it is necessary to make a photometric correction for the detected sources. For this reason we will correct all the data acquired in the NUV and visual regimes using the Anderson \& Bedin algorithm. 


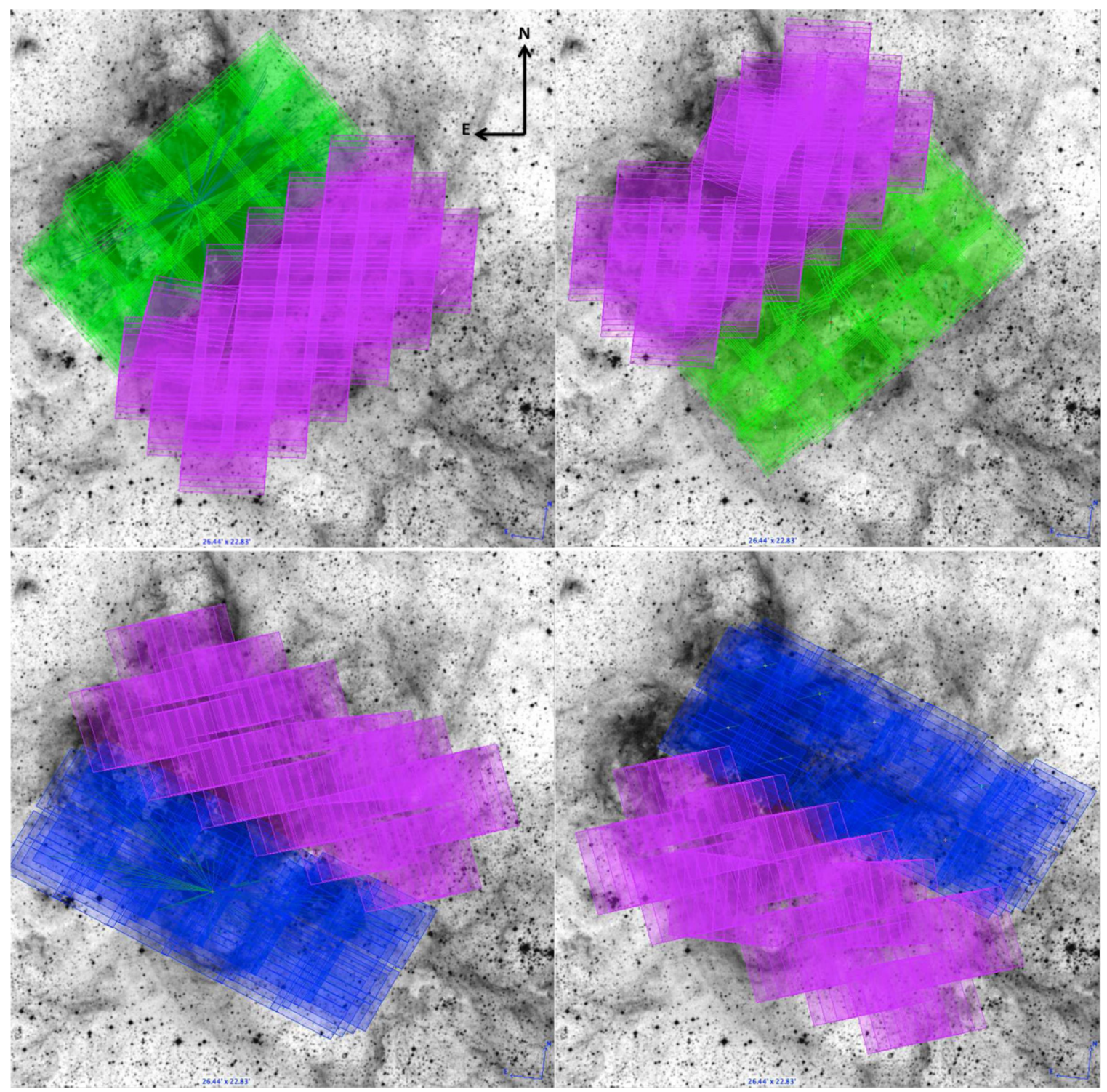

Figure 5. Upper panels: positions of the HST pointings in the filters F110W, F160W (WFC3) and F658N (ACS), superimposed on the $33^{\prime} \times 21^{\prime}$ DSS image of 30 Doradus. WFC3 is in green and ACS in magenta. Lower panels: positions of the HST WFC3 (F275W and F336W filters) and ACS (filter F555W) pointings. WFC3 is in blue and ACS is in magenta. Figure 1 is rotated by $45^{\circ}$ with the respect to these plots.

(A color version of this figure is available in the online journal.)

\section{DATA ANALYSIS}

The analysis of the F775W (GO-12499) and the NIR data was carried out directly on the bias-subtracted and flat-fielded exposures processed by the standard $H S T$ calibration pipelines CALACS and CALWF3. The outputs from the HST calibration pipelines are still in the raw-detector pixel frame and thus retain several kinds of spatial distortions that have to be taken into account.

\subsection{Preparation of the Reference Frame}

Observations in the F775W filters cover a projected area of $\sim 14^{\prime} \times 12^{\prime}$. The first step in reducing the data was to create a distortion-free reference frame and relate the astrometry and photometry of each exposure to this frame. To achieve this, in each F775W deep exposure we measured positions and fluxes for all the sources that had more than 100 counts in each exposure and no brighter neighbors within a 5 pixels radius using the program img2xym_WFC.09x10 (Anderson \& King
2006). ${ }^{20}$ This program uses libraries of empirical point-spread functions (PSFs) to account for the spatial variations caused by the optics of the telescope and the variable charge diffusion in the CCD and creates a geometric-distortion corrected list of sources.

We used the Two Micron All Sky Survey (2MASS) cata$\log$ (Skrutskie et al. 2006) as an initial astrometric reference frame. We then found common stars between each image list and 2MASS to define the general six-parameter linear transformation between the distortion-corrected frame of each exposure into the 2MASS-defined frame. We used these transformations to collate the individual star lists in the reference frame and determine the average position for $\sim 110,000$ bright, isolated, unsaturated stars that in the deep exposures have three or more coincident detections. 20 The program was originally designed for ACS/WFC data, but versions for
both the WFC 3 channels are now available. 
For the ACS data we initially used the geometric distortion and PSF library of Anderson \& King (2006). Both the PSF and distortion solution were determined using data acquired before the last Hubble servicing mission (SM4). A careful inspection of the position residuals showed that the PSF has developed a $2 \%$ asymmetry and the distortion solution has changed by $\sim 4 \%$ of a pixel since SM4. Although this is a very small change, it is sufficient to introduce noticeable errors in very wide mosaics, therefore we used all the bright and isolated stars found in the ACS field of view to derive a new PSF and update the geometric distortion. Since all the observations in this program were acquired with very similar rotation angle, we were not able to independently solve for the ACS skew term, however, we used the common stars between ACS and WFC3 to calibrate it relative to WFC3.

Finally we improved the internal quality of the reference frame by iteration. The rms residuals of the average positions were less than 0.01 pixel in each coordinate. In this process we have mapped the ACS pixel scale to the size of the WFC3 UVIS channel, so that in all the catalogs a pixel corresponds to 0.040 . The final reference list was then used to compute the final astrometric transformation of each exposure (for both ACS and WFC3) into the reference frame.

\subsection{The Source-finding Routine}

The stars used in Section 4.1 to align the single exposures to the reference frame were found in a single pass through each image. This approach will find almost all the bright stars in a field, but will miss many of the obvious faint sources, especially those in the wings of bright objects.

To identify the fainter sources and push the photometry as deep as possible, we used an evolution of the program described in Anderson et al. (2008). The new program will be fully described in a forthcoming paper (J. Anderson et al., in preparation).

The previous version of the code was designed to work only with ACS and could handle only two filters. This new routine can handle multiple instruments and several filters at the same time. The routine first finds the bright stars, then subtracts them and searches for fainters stars in the subtracted images. It performs several such finding iterations (Figure 6), using detection thresholds that depend on the number of exposures available at each point in the field. This approach is particularly useful when, as in the case of our survey, the coverage is not uniform (in some regions we have only one or two exposures, while in others we have up to 14 overlapping exposures, Figure 4). An inspection by eye shows that the code efficiently avoids spurious detections caused by the diffraction spikes of saturated stars and sharp gaseous filaments (Figure 6).

We were able to identify more than $1.1 \times 10^{6}$ sources in the F775W observations. The photometry was calibrated to the VEGAmag photometric system using the zero points listed on STScI Web site. ${ }^{21}$ These zero points are derived for images combined using "astrodrizzle," thus they cannot be directly applied to our flt-based photometric catalogs. To calibrate the flt-based catalogs into the VEGAmag system we selected several isolated bright stars in the drizzled images and measured their magnitudes using 0.'5 aperture photometry for ACS and 0.'4 for WFC3 data and applied the appropriate zero point. For these stars we then determined the difference between the flt-based

\footnotetext{
21 http://www.stsci.edu/hst/wfc3/phot_zp_lbn and http://www.stsci.edu/hst/acs/analysis/zeropoints
}

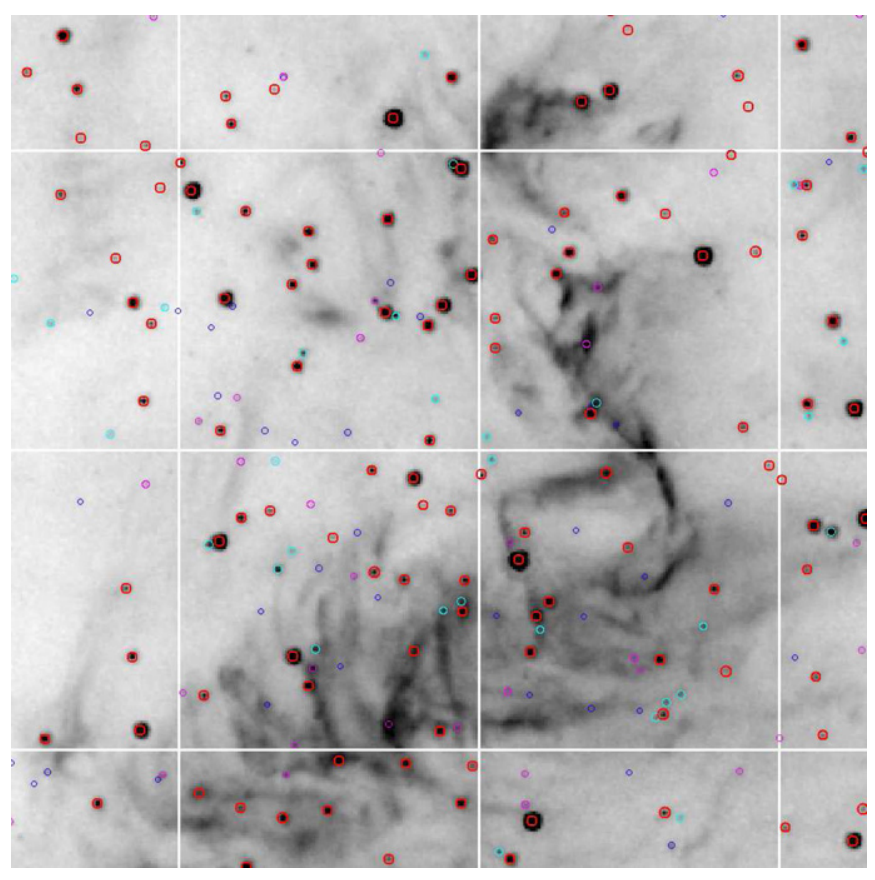

Figure 6. Stars found in a $360 \times 360$ pixel region in the F775W filter. Sharp filaments and unresolved knots of gas are visible. Different colors and sizes of the circles correspond to different finding iterations in the photometry.

(A color version of this figure is available in the online journal.)

magnitude and the magnitude in the VEGAmag system and used the average differences as the final zero points.

The F775W photometric catalog was used to measure the sources in the IR images. Of the initial $1.1 \times 10^{6}$ sources, $\sim 480,000$ are in the region observed with the first block of IR data.

In addition to finding and measuring stars, the reduction routine also produces several diagnostics to help identify the stars that are well-measured. One such parameter is Q, which reports the linear-correlation coefficient between the PSF and the stellar image: $Q=1.0$ is a perfect fit. This parameter is particularly useful in identifying unresolved objects such as background galaxies and blended sources. We also used the rms of the individual photometric measurements about the mean to help identify well measured stars.

In both ACS and WFC3 images acquired with the F775W filters, stars brighter than $m_{\mathrm{F} 775 \mathrm{~W}}=15$ are saturated even in the shorter exposures. As a result the $Q$ parameter for these stars differs significantly from 1 . We used aperture photometry to recover the flux of these sources, and we did not apply any selection criteria. For ACS we then selected the sources in the magnitude range between $15 \leqslant m_{\mathrm{F} 75 \mathrm{~W}_{\mathrm{ACS}}}<21$ with $\mathrm{Q}>$ 0.95 , while for sources fainter than $m_{\mathrm{F} 775 \mathrm{~W}_{\mathrm{ACS}}} \geqslant 21$ we selected only those objects with $Q>0.92$ or photometric error $<0.5$. Similarly, for the F775W WFC3 filter in the magnitude range between $15 \leqslant m_{\mathrm{F} 775 \mathrm{~W}_{\mathrm{WFC} 3}}<22$ we selected only the objects with $Q>0.95$ and for fainter sources the photometric error had to be better then 0.5 .

Because of the coarser resolution of the IR channel, crowding is more severe in the filters F110W and F160W and the parameter $Q$ does not provide an estimate of the quality of the photometry that is as good as in the case of the optical wavelengths. Moreover, for most of the field in the NIR we have only two measurements per filter, therefore to estimate the photometric error we used the signal to noise instead of the 

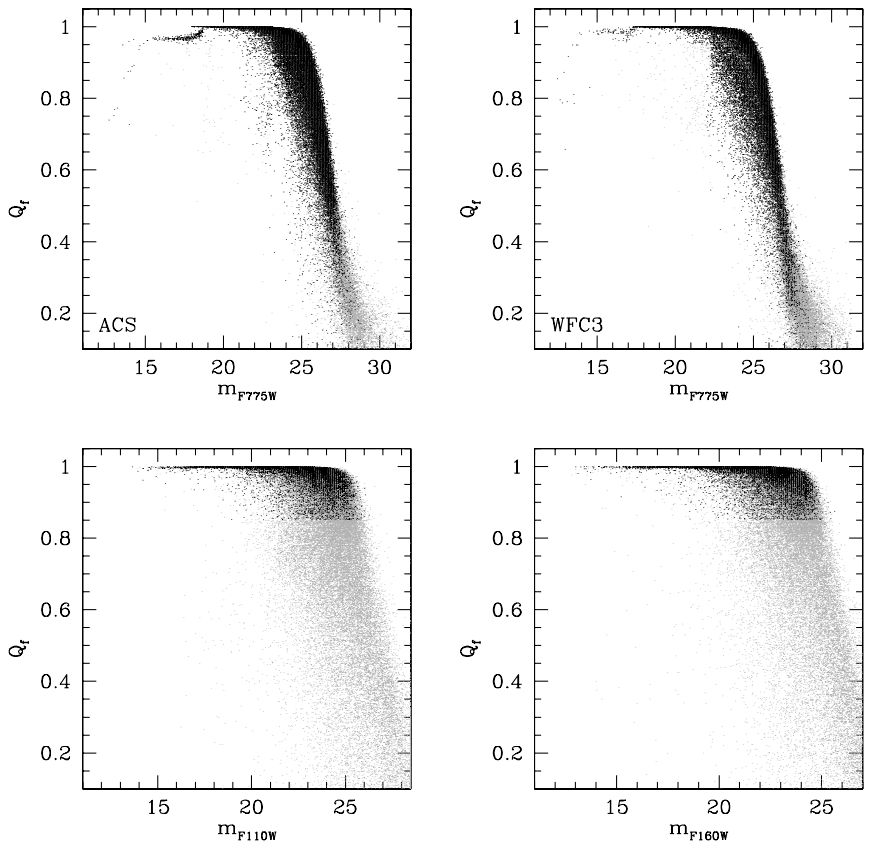

Figure 7. From the top to the bottom, clockwise, Q parameters as a function of magnitude for the filters F775W (ACS and WFC3 respectively), F160W and F110W. Only $10 \%$ of all the sources detected in the F775W filers and $20 \%$ of the sources found in the F110 and F160W filters are shown. Objects that meet the selection criteria are in black.

rms of the individual photometric measurements. As a result for both the F110W and F160W filters we selected only those objects with $Q>0.85$ and photometric error smaller than 0.1 . After these selections the combined ACS + WFC3 catalog in the F775W filter contains more than 366,000 stars, of which $\sim 150,000$ that are detected in both the F110W and F160W filters. Figure 7 shows $Q$ as a function of magnitude for the two F775W filters (ACS and WFC3 are showed separately) and for the NIR data. Sources that meet the selection criteria are shown in black, while the remaining sources are in gray.

\section{Color-Magnitude and Color-Color Diagrams}

Figure 8 shows the Hess CMDs and CCD for different combinations of the F775W, F110W, and F160W filters. The main evolutionary features are highlighted for reference in the top left-panel. In this CMD stars brighter than $m_{\mathrm{F} 110 \mathrm{~W}} \sim 20$ and bluer than $m_{\mathrm{F} 775 \mathrm{~W}}-m_{\mathrm{F} 110 \mathrm{~W}} \sim 0.5$ are in the upper main sequence (UMS). These are intermediate- and high-mass stars. The UMS is representative of a stellar population younger than $\sim 1 \mathrm{Gyr}$.

Stars fainter than $m_{\mathrm{F} 110 \mathrm{~W}} \sim 22$ and bluer than $m_{\mathrm{F} 775 \mathrm{~W}}-$ $m_{\mathrm{F} 110 \mathrm{~W}} \sim 1$ are in the lower main sequence (LMS). This is a mixture of stars with ages spanning the age range from potentially very young to several gigayears old.

Stars brighter than $m_{\mathrm{F} 110 \mathrm{~W}} \sim 21$ and redder than $m_{\mathrm{F} 775 \mathrm{~W}}-$ $m_{\mathrm{F} 110 \mathrm{~W}} \sim 0.5$ are in the sub giant branch (SGB), RGB and red clump $(\mathrm{RC})$ evolutionary phases. These sources are characteristic of an evolved old ( $\gtrsim 1-2 \mathrm{Gyr})$ stellar population. In all the CMDs, the $\mathrm{RC}$ is elongated, extending over more than $\sim 1.1 \mathrm{mag}$ in the F110W filter. Similarly both the UMS and the RGB are broad suggesting that the reddening is variable and differential extinction often is significant across the field.

The lower $\left(m_{\mathrm{F} 110 \mathrm{~W}} \gtrsim 21\right)$ right $\left(m_{\mathrm{F} 775 \mathrm{~W}}-m_{\mathrm{F} 110 \mathrm{~W}} \gtrsim 1\right)$ corner of the CMD is usually populated by young, intermediate- and low-mass PMS stars. These sources have not yet started the hydrogen burning in their core and are still contracting toward the zero age main sequence.

In other young $(\lesssim 5 \mathrm{Myr}$ old) clusters in the Magellanic Clouds, the PMS stars are normally well separated from the LMS stars in the CMDs derived from HST observations. This is not the case for the CMDs shown in Figure 8 not only because differential reddening shifts LMS stars toward redder colors, but also because with the aging of the stellar population PMS stars move toward bluer colors. As the presence of the $\sim 20-25 \mathrm{Myr}$ old cluster Hodge 301 indicates, star formation in the Tarantula Nebula region has been active for several Myr, therefore in the CMDs of the entire field of view, LMS and PMS are overlapping, however, when smaller regions, centered on recent (i.e., <5 Myr) episodes of star formation are examined, the separation between LMS and PMS becomes more evident (Figure 9).

\subsection{Spatial Distribution of the Stellar Populations}

To better understand the spatial distribution of the identified stellar populations, and at the same time, highlight how the reddening changes as a function of position, we have divided our catalog into 18 regions of $3000 \times 3000$ pixel each, corresponding to $\sim 29 \mathrm{pc} \times 29 \mathrm{pc}$. Figure 9 shows the location of these regions on the F160W mosaic (upper panel) and the corresponding $18 m_{\mathrm{F} 110 \mathrm{~W}}$ versus $m_{\mathrm{F} 110 \mathrm{~W}}-m_{\mathrm{F} 160 \mathrm{~W}} \mathrm{CMDs}$ (lower panel).

The inspection of these CMDs shows that the UMS is always well populated, with the exception of regions A, B, D, G, Q, and $\mathrm{R}$. These six regions are likely sampling the field of the LMC.

The majority of R136 is in region I, and the NE clump identified by Sabbi et al. (2012) is in region K. The CMDs for both these regions show a large number of red and faint PMS stars, as well as very well populated UMSs. Both R136 and the NE clump are very young systems ( $\leqslant 5 \mathrm{Myr})$, and the PMS stars are well separated from the LMS stars. PMS stars are likely also present in regions $\mathrm{L}$ and $\mathrm{N}$.

Hodge 301 is in region O. The UMS of the corresponding CMD is well populated in agreement with the young age of the cluster. At these ages PMS stars are too blue to be distinguished from the LMS stars using broad-band photometry only.

The RC and the RGB vary from relatively compact and well defined (as in regions $\mathrm{B}, \mathrm{E}, \mathrm{M}, \mathrm{O}, \mathrm{Q}$, and $\mathrm{R}$ ) to extremely broad. For example in region $\mathrm{J}$, the $\mathrm{RC}$ covers more than 4 mag in the F110W filter, a typical signature of differential reddening. In their analysis of the reddening in the LMC, based on the RC, Haschke et al. (2011) find that not only 30 Dor exhibits the highest reddening of any region in this galaxy, but also the highest differential reddening.

It is interesting to note that young and old stars are affected by different amounts of extinction, with the reddening being on average less severe for the young stars, than the old ones. This effect is particularly evident for example in region $\mathrm{H}$, where the UMS is narrow and well defined, but the magnitude of the $\mathrm{RC}$ ranges between $18 \lesssim m_{\mathrm{F} 110 \mathrm{w}} \lesssim 20$. This is apparently at variance with conclusions by Zaritsky (1999), who found that on average in the LMC old stars are less obscured by dust than hot young stars.

In deriving the reddening map, Zaritsky (1999) used only those stars that were detected at the same time in the four bands $U, B, V$, and $I$. This selection criterion penalizes the most extinguished evolved stars, therefore the average amount of reddening derived for the RGB stars may have been underestimated. As shown in Haschke et al. (2011) in these highly extinguished regions the distribution of the reddening values is not Gaussian, but shows an extended tail toward the higher values. 

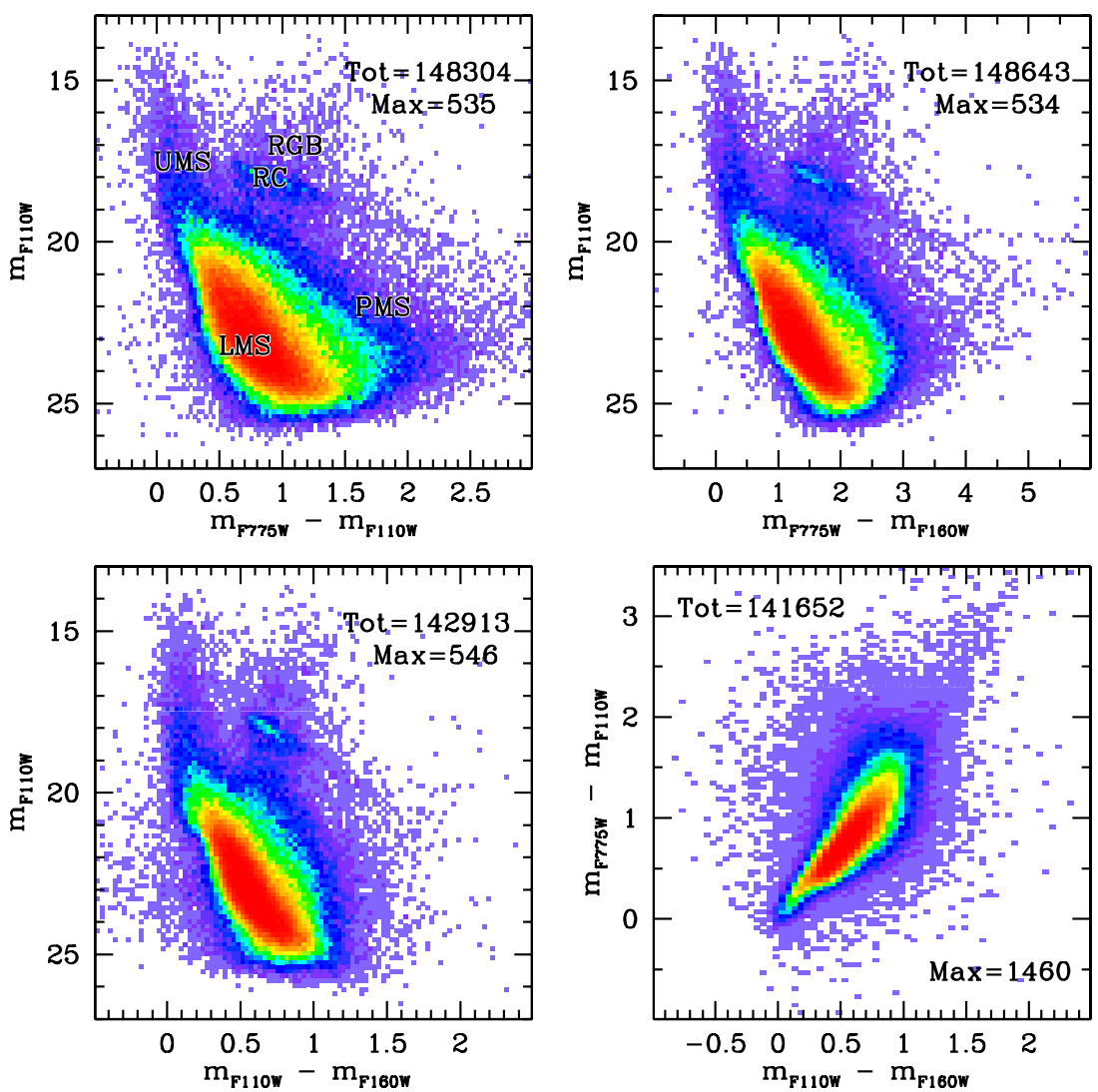

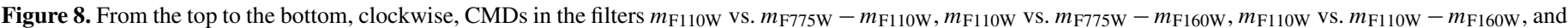

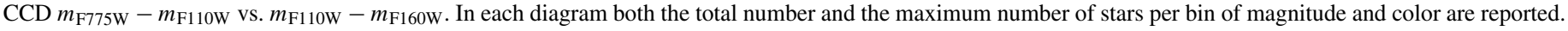
The most important evolutionary sequences are highlighted in the upper-left panel for reference.

(A color version of this figure is available in the online journal.)

It has to be noted that most of the UMS stars found in the Tarantula Nebula region are associated with the starburst that originated R136 and can be all considered to be at the same distance from us. The RGB stars, on the other hand, are evenly distributed in the entire body of the LMC and are more affected by the three-dimensional structure of the galaxy. If for example 30 Dor is on the nearer side of the LMC disk, the majority of the RGB stars in our field would be beyond 30 Dor and will be extinguished by the dust associated to the Tarantula Nebula. We will be able to better characterize this issue when we observe the region in bluer filters (F275W, F336W, and F555W).

The CMDs in Figure 9 suggest that stellar populations of different ages are not uniformly distributed. To better highlight this effect, in Figure 10 we have compared the spatial distribution of stars selected from different evolutionary sequences.

In panel (A) we have selected UMS stars brighter than $m_{\mathrm{F} 110 \mathrm{~W}}<16.6$, and bluer than $m_{110 \mathrm{w}}-m_{160 \mathrm{w}}<0.3$. These stars are younger than $\sim 20 \mathrm{Myr}$ and are mostly concentrated around R136 and the NE clump. The IR observations collected so far cover 9 (namely, 537, 470, 398, 385, 382, 392, 706, 682, and 581) of the $15 \mathrm{O}$ stars identified by Bressert et al. (2012) as candidate high-mass stars formed in isolation, and another 5 targets will be observed in 2013 July. As already reported by these authors, there is no evidence for an underlying cluster around these sources. Further constraints on the true nature of these stars will come when proper motion measurements will be available.

Panel (B) shows the distribution of stars in the magnitude range between $17.9<m_{\mathrm{F} 110 \mathrm{~W}}<19.9$, and bluer than $m_{\mathrm{F} 110 \mathrm{~W}}-$
$m_{\mathrm{F} 160 \mathrm{~W}}<0.4$. At the distance of the LMC this correspond to a mass range between $\sim 3$ and $\sim 7 M_{\odot}$. After $\sim 2 \mathrm{Myr}$ the most massive PMS stars in this mass range are already merging into the UMS, while a $\sim 3 M_{\odot}$ star will still be on the MS after $\sim 150$ Myr (Dell'Omodarme et al. 2012). Therefore panel (B) shows the distribution of stars in the age range between $\sim 2$ and 150 Myr. R136 and the NE clump are still very well defined. The cluster to the northwest of the image is Hodge 301. A careful inspection of panel (A) shows that Hodge 301 is also visible in that map.

Sources in panel (C) have colors between $0.7<m_{\mathrm{F} 110 \mathrm{~W}}-$ $m_{\mathrm{F} 160 \mathrm{~W}}<1.3$ and magnitudes between $20.5<m_{\mathrm{F} 110 \mathrm{~W}}<22.5$. This is the locus of $\lesssim 1 M_{\odot}$ PMS stars. These sources are likely younger than $\sim 5$ Myr. Hodge 301 is not visible in this region, while a "chain" of young clustered objects connects the NE clump to the northern corner of the map. Some of these clusters coincide with the embedded massive $\mathrm{O}$ stars found in the dense knots of dust at the interface between R136 and Hodge 301, already identified by Brandner et al. (2001). Others are aligned with one side of the shell of ionized gas that surrounds a soft and bright X-ray-emitting bubble (Meaburn 1984; Wang \& Helfand 1991; Townsley et al. 2006). Some of these clusters likely host embedded OB stars (Walborn et al. 2013). Although it is possible that the feedback from the two massive clusters NGC 2070 and Hodge 301 may have triggered the star formation in the nearer clumps, the majority of the clusterings are at a projected distance from R136 between 30 and $60 \mathrm{pc}$, too far away to be affected by R136 in the less then $\sim 2 \mathrm{Myr}$. Alternatively, these systems may result from the collapse of residual pockets 

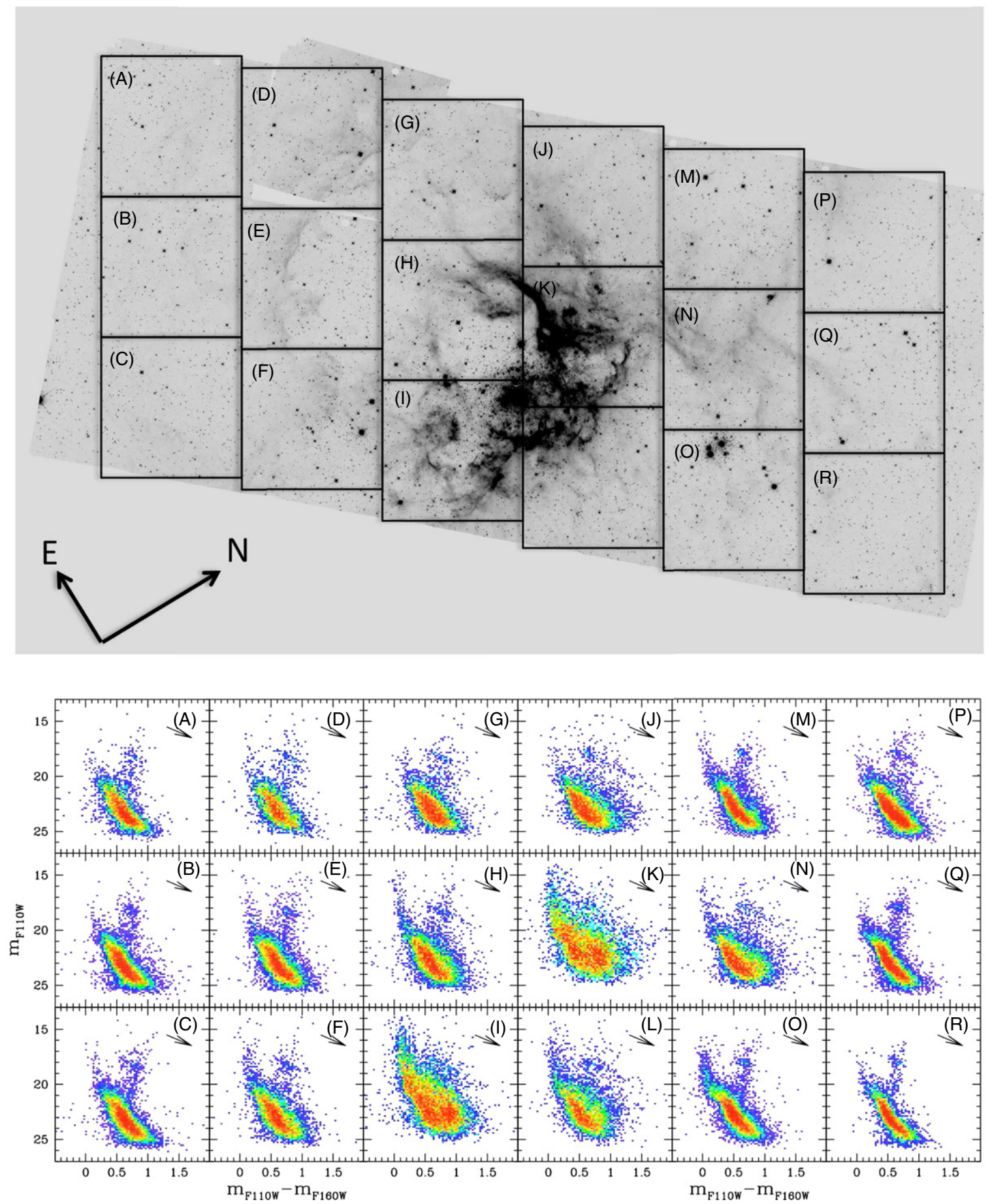

Figure 9. Upper panel: mosaic of the images acquired in the F160W filter. The mosaic has been divided into 18 regions of $\sim 3000 \times 3000$ pixels. The corresponding $18 m_{\mathrm{F} 110 \mathrm{~W}}$ vs. $m_{\mathrm{F} 110 \mathrm{~W}}-m_{\mathrm{F} 160 \mathrm{~W}} \mathrm{CMDs}$ are shown in the lower panel.

(A color version of this figure is available in the online journal.)

of gas formed during the fragmentation of the giant molecular cloud that formed R136, as predicted by the models that invokes fractal initial conditions and assembling of sub-clusters to form massive young clusters (Aarseth \& Hills 1972; McMillan et al. 2007; Allison et al. 2009; Moeckel \& Bonnell 2009; Bonnell et al. 2011; Smith et al. 2011; Fuji et al. 2012; Fujii \& Portegies Zwart 2013).

LMS stars (panel (D)) have been selected in the color range $0.26<m_{\mathrm{F} 110 \mathrm{~W}}-m_{\mathrm{F} 160 \mathrm{~W}}<0.65$ and magnitudes between $21.5<m_{\mathrm{F} 110 \mathrm{~W}}<22.5$. These sources have ages between a few tens of megayears and several gigayears. The spatial distribution of these sources is quite uniform in agreement with our assumption that we are looking at the stars in the field of the LMC. The only visible cluster in panel (D) is Hodge 301, in agreement with the fact that old PMS stars cannot be separated from LMS stars using broad-band photometry only. R136 and the NE clump are not visible in this map, as expected from their very young $(<5 \mathrm{Myr})$ age.

The number of LMS stars is significantly lower in the regions that in panel $(\mathrm{C})$ correspond to sites of very recent 

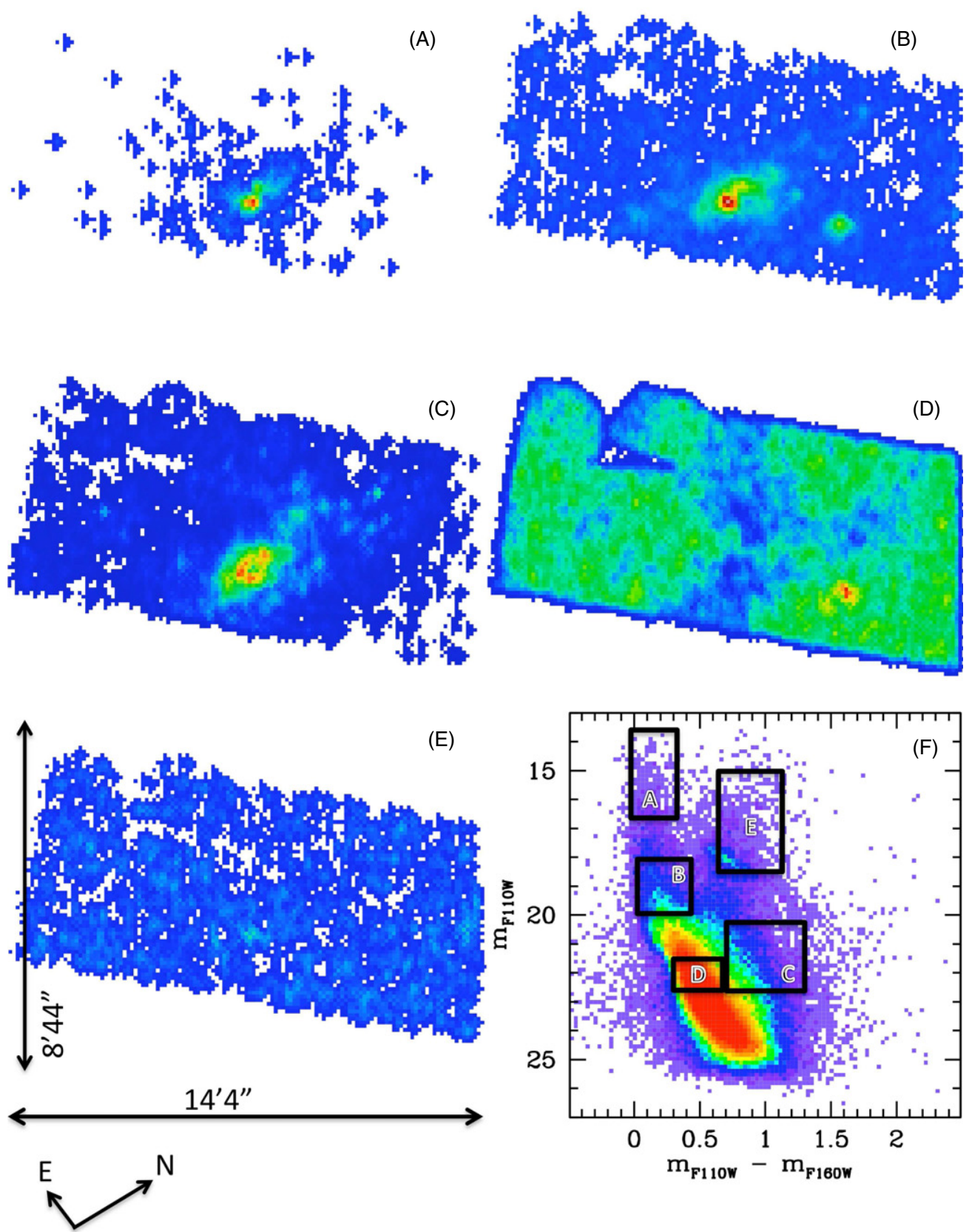

Figure 10. Spatial distribution of the massive UMS stars (panel (A)), intermediate mass stars in the UMS (panel (B)), PMS stars (panel (C)), LMS stars (panel (D)) and RGB stars (panel (E)). The regions of the $m_{\mathrm{F} 110 \mathrm{~W}}$ vs. $m_{\mathrm{F} 110 \mathrm{~W}}-m_{\mathrm{F} 160 \mathrm{~W}}$ CMD used to select the stellar populations are shown in panel (F).

(A color version of this figure is available in the online journal.)

star formation. These regions are characterized by a higher stellar density and nebulosity, making it harder to detect faint stars, and thus the low number of LMS may be the result of a lower level of completeness. We note that many of these regions are obscured by dust. If the Tarantula Nebula is on the nearer side of the LMC disk, the dust associated to the star-forming region would attenuate the luminosity of the LMS, pushing them outside the range of magnitudes and colors that we are considering. Artificial star tests and stellar SED fitting will allow us to better discriminate between these two hypotheses. The spatial distribution of RGB stars (panel (E), $\left.0.6<m_{\mathrm{F} 110 \mathrm{~W}}-m_{\mathrm{F} 160 \mathrm{~W}}<1.1,15.3<m_{\mathrm{F} 110 \mathrm{~W}}<18.5\right)$ is noisy because of low number statistics, but on average appears quite uniform.

\section{DISCUSSION AND CONCLUSIONS}

We have discussed the observing strategy and presented preliminary results from the first half of the IR observations of the "Hubble Tarantula Treasury Project (HTTP: Unraveling Tarantula's Web)," an ongoing panchromatic HST survey designed to resolve and characterize the stellar populations in the Tarantula Nebula down to the sub-solar mass regime $\left(<0.5 M_{\odot}\right)$. HTTP is collecting deep observations of the Tarantula Nebula in the NUV (F257W and F336W), optical (F555W and F658N), and NIR (F110W and F160W). These data are combined with deep archival observations of the region in the F775W filter.

The analysis of the data acquired this far indicates that the reddening is highly variable across the region, with the young 
UMS stars being on average less extinguished by dust than the old RGB stars. This could be a sign that 30 Dor is on the nearer side of the LMC disk. Because the extension of 30 Dor is likely negligible with respect to the LMC depth, the majority of the evolved stars could be beyond 30 Dor, and therefore their luminosity would be extinguished by the dust associated with the Tarantula Nebula.

We find that stellar populations of different ages have very different spatial distributions. While the intermediate- and old-age stars have had time to diffuse in the disk of the LMC, the younger stars are still associated with their birth sites. In particular the majority of the UMS stars are associated with R136, the NE clump and Hodge 301.

An inspection of the spatial distribution of the PMS stars reveals the presence of several small clustered systems. The majority of these systems are located between R136 and the northern corner of the area covered by our survey, along the ridge of an X-ray-emitting bubble. We speculate that these systems may be minor episodes of star formation that occurred during the fragmentation of the giant molecular cloud that formed R136. Our preliminary analysis confirms that the region has actively formed stars for $\sim 20 \mathrm{Myr}$ and possibly longer. Once the NUV and optical data are available, we will use a Bayesian SEDfitting algorithm to derive the star-by-star reddening correction and constrain the age of the various sites of recent star formation. A comparison of our data with synthetic CMDs will allow us to better assess how star formation occurred and is propagating in the Tarantula Nebula, and at what star formation rate.

The uniform coverage and the broad selection of filters of HTTP will benefit the community in the following ways.

1. $\mathrm{H} \alpha$ and NIR images will make it possible to identify emission-line stars such as Be stars, RGB and SGB stars with stellar winds, Herbig Ae/Be stars, and low mass PMS stars.

2. Super star clusters such as R136 are often considered present-day counterparts of forming globular clusters. HTTP offers an observational snapshot of the early evolutionary phases of these systems and new information on how they relate to their surroundings during early evolutionary phases.

3. HTTP will yield the richest and most homogenous sample of moderately metal-poor PMS stars of different ages and masses. SEDs and mass-accretion rates, which can be derived from this dataset, will provide new and much needed constraints on the evolutionary models of PMS stars.

4. HTTP can be used to measure the scale at which UV radiation from hot massive stars affects the evolution of low-mass accreting PMS stars.

HTTP is a treasury program and all the data are immediately available to the public and can be downloaded from the MAST archive. In addition we will deliver a series of high-level products to the community, including the following.

1. A unified star catalog for all the filters (F275W, F336W, F555W, F658N, F775W, F110W, and F160W). For each filter we will provide an average flux and an error in flux, based on multiple independent observations.

2. Artificial star tests done with stars inserted along the fiducial sequences. This will allow users to locally quantify the level of completeness of the catalog and the extent to which crowding may have broadened the sequences.
3. Co-registered stacked images of the field in all filters, with calibrated WCS headers.

4. Maps of differential reddening and the star formation history of the field.

5. A catalog with the properties of all the clusters and associations.

At the moment, only a few large ground-based telescopes $(6 \mathrm{~m}+)$ can surpass the sharpness of Hubble's data at certain wavelengths and on a very limited field of view. In the near future ALMA and the James Webb Space Telescope (JWST) will have spatial resolution and sensitivity similar to HST, but because of the different wavelength coverage, they will probe complementary but distinct stellar populations and evolutionary stages. In particular JWST and ALMA will be able to identify the most dust-embedded young stellar objects, highlighting where the most massive stars are now forming. In summary, once combined with the our ongoing investigations (i.e., stellar dynamics, PI: Lennon; gas dynamics, PI: Gallagher) and studies at other wavelengths, HTTP will provide a complete high-resolution picture of the complex interplay between stars, gas, dust and stellar feedback in a bursting regime. HTTP will become the definitive catalog of the field and it will provide a unique way to cross-identify objects in multiple studies, serving as a touchstone for all future works on 30 Dor in particular, and on starbursts in general.

The authors are grateful to Zolt Levay for his work on the images shown in Figures 1 and 3. M.T. and M.C. have been partially funded by contracts ASI I009/10/0, PRININAF-2010 and PRIN-MIUR-2010-11. Support for programs GO-12499 and GO-12939 was provided by NASA through grants from the Space Telescope Science Institute, which is operated by the Association of Universities for Research in Astronomy, Inc., under NASA contract NAS 5-26555. E.K.G. acknowledges support from the Collaborative Research Center "The Milky Way System" (SFB 881) of the German Research Foundation (DFG), particularly by subproject B5. D.A.G. kindly acknowledges financial support by the German Research Foundation (DFG) through grant GO 1659/3-1. S.d.M. acknowledges support by NASA through Hubble Fellowship grant HST-HF-51270.01-A awarded by the Space Telescope Science Institute, which is operated by the Association of Universities for Research in Astronomy, Inc., for NASA, under contract NAS 5-26555.

\section{REFERENCES}

Aarseth, S. J., \& Hills, J. G. 1972, A\&A, 21, 255

Allison, R. J., Goodwin, S. P., Parker, R. J., et al. 2009, ApJL, 700, L99

Andersen, M., Zinnecker, H., Moneti, A., et al. 2009, ApJ, 707, 1347

Anderson, A., \& King, J. R. 2006, STSCI Institute Science Report ACS 2006-01 (Baltimore, MD: STScI)

Anderson, J., \& Bedin, L. R. 2010, PASP, 122, 1035

Anderson, J., Sarajedini, A., Bedin, L. R., et al. 2008, AJ, 135, 2055

Annibali, F., Greggio, L., Tosi, M., Aloisi, A., \& Leitherer, C. 2003, AJ, 126,2752

Annibali, F., Tosi, M., Monelli, M., et al. 2009, AJ, 138, 169

Baggett, S., \& Anderson, J. 2012, STScI Institute Science Report WFC3 2012-12

Bonnell, I. A., Smith, R. J., Clark, P. C., \& Bate, M. R. 2011, MNRAS, 410, 2339

Brandl, B. R., Devost, D., Higdon, S. J. U., et al. 2004, ApJS, 125, 188

Brandner, W., Grebel, E. K., Barbá, R. H., Walborn, N. R., \& Moneti, A. 2001, AJ, 122, 858

Bressert, E., Bastian, N., Evans, C. J., et al. 2012, A\&A, 542, A49

Chen, Y., Wang, Q. D., Gotthelf, E. V., et al. 2006, ApJ, 651, 237 
Chu, Y.-H., \& Kennicutt, R. C., Jr. 1994, AJ, 108, 1696

Cignoni, M., Tosi, M., Sabbi, E., et al. 2010, ApJL, 712, L63

Crowther, P. A., Schnurr, O., Hirschi, R., et al. 2010, MNRAS, 408, 731

Dale, J. E., Ercolano, B., \& Bonnell, I. A. 2012, MNRAS, 427, 2852

Dekel, A., \& Silk, J. 1986, ApJ, 303, 39

de la Caille, N. L. 1761, RSPT, 52, 21

Dell'Omodarme, M., Valle, G., Degl'Innocenti, S., \& Prada Moroni, P. G. 2012 A\&A, 540A, 26

De Marchi, G., Panagia, N., \& Romaniello, M. 2010, ApJ, 715, 1

De Marchi, G., Paresce, F., Panagia, N., et al. 2011, ApJ, 739, 27

Douglas, L. S., Bremer, M. N., Lehnert, M. D., \& Stanway, E. R. 2010, MNRAS, 409, 1155

Elmegreen, B. G., \& Lada, C. J. 1977, ApJ, 214, 725

Evans, C. J., Taylor, W. D., Hénault-Brunet, V., et al. 2011, A\&A, 530, A108

Evans, C. J., Walborn, N. R., Crowther, P. A., et al. 2010, ApJL, 715, L74

Feast, M. W. 1953, Obs, 73, 255

Feitzinger, J. V., Schlosser, W., Schmidt-Kaler, T., \& Winkler, C. 1980, A\&A, 84,50

Ferguson, H., \& Babul, A. 1998, MNRAS, 296, 585

Fujii, M. S., \& Portegies Zwart, S. 2013, MNRAS, 430, 1018

Fujii, M. S., Saitoh, T. R., \& Portegies Zwart, S. F. 2012, ApJ, 753, 85

Gouliermis, D. A., Schmeja, S., Dolphin, A. E., et al. 2012, ApJ, 748, 64

Grebel, E. K. 1997, A\&A, 317, 448

Grebel, E. K., \& Chu, Y.-H. 2000, AJ, 111, 787

Haschke, R., Grebel, E. K., \& Duffau, S. 2011, AJ, 141, 158

Heckman, T. M. 2005, in Starbursts: From 30 Doradus to Lyman Break Galaxies, ed. R. de Grijs \& R. M. González Delgado (Astrophysics and Space Science Library, vol. 329; Dordrecht: Springer), 3

Heckman, T. M., Kauffmann, G., Brinchmann, J., et al. 2004, ApJ, 613, 109

Herschel, J. F. W. 1847, Results of Astronomical Observations Made during the Years 1834, 5, 6, 7, 8, at the Cape of Good Hope; Being the Completion of a Telescopic Survey of the Whole Surface of the Visible Heavens, Commenced in 1825 (London: Smith, Elder, \& Co.)

Hunt, L. K., \& Hirashita, H. 2009, A\&A, 507, 1327

Hunter, D., O’Neil, E. J., Lynds, R., et al. 1996, ApJL, 459, L27

Hunter, D., Shaya, E. J., Holtzman, J. A., et al. 1995, ApJ, 448, 179

Hunter, D. A., \& Elmegreen, B. G. 2006, ApJS, 162, 49

Kennicutt, R. C., \& Evans, N. J. 2012, ARA\&A, 50, 531

Kennicutt, R. C., \& Hodge, P. W. 1986, ApJ, 306, 130

Konstantopoulos, I. S., Bastian, N., Smith, L. J., et al. 2009, ApJ, 701, 1015

Lee, J. C., Gil de Paz, A., Tremonti, C., et al. 2009, ApJ, 706, 599

Lee, J. C., Kennicutt, R. C., Funes, S. J., et al. 2007, ApJL, 671, L113

Leitherer, C. 1998, in Stellar Astrophysics for the Local Group: VIII Canary Islands Winter School of Astrophysics, ed. A. Aparicio, A. Herrero, \& F. Sanchez (Cambridge: Cambridge Univ. Press), 527

MacKenty, J. W., \& Smith, L. J. 2012, CTE White Paper, Space Telescope Science Institute, http://www.stsci.edu/hst/wfc3/ins_performance/CTE/CTE_ White_Paper.pdf
Mac Low, M.-M., \& Ferrara, A. 1999, ApJ, 513, 142

McMillan, S. L. W., Vesperini, E., \& Portegies Zwart, S. F. 2007, ApJL, $655, \mathrm{~L} 45$

McQuinn, K. B. W., Skillman, E. D., Cannon, J. M., et al. 2012, ApJ, 759, 77

McQuinn, K. B. W., Skillman, E. D., Dalcanton, J. J., et al. 2010, ApJ, 724, 49

Meaburn, J. 1984, MNRAS, 211, 521

Meurer, G. R., Heckman, T. M., Lehnert, M. D., Leitherer, C., \& Lowenthal, J. 1997, AJ, 114, 54

Moeckel, N., \& Bonnell, I. A. 2009, MNRAS, 400, 657

Moffat, A. F. J. 1982, JRASC, 76, 323

Nikolaev, S., Drake, A. J., Keller, S. C., et al. 2004, ApJ, 601, 260

O'Connell, R. W. 2005, in Starbursts: From 30 Doradus to Lyman Break Galaxies, ed. R. de Grijs \& R. M. Gonzàlez Delgado (Astrophysics and Space Science Library, vol. 329; Dordrecht: Springer), 333

O’Connell, R. W., Gallagher, J. S., III, Hunter, D. A., \& Colley, W. N. 1995, ApJL, 446, L1

O’Connell, R. W., \& Mangano, J. J. 1978, ApJ, 221, 62

Oey, M. S., Parker, J. S., Mikles, V. J., \& Zhang, X. 2003, AJ, 126, 2317

Panagia, N., Gilmozzi, R., Macchetto, F., Adorf, H. M., \& Kirshner, R. P. 1991, ApJL, 380, L23

Panagia, N., Romaniello, M., Scuderi, S., \& Kirshner, R. P. 2000, ApJ, 539, 197

Pietrzyński, G., Graczyk, D., Gieren, W., et al. 2013, Natur, 495, 76

Romaniello, M., Panagia, N., Scuderi, S., \& Kirshner, R. P. 2002, AJ, 123,915

Sabbi, E., Lennon, D. J., Gieles, M., et al. 2012, ApJL, 754, L37

Sabbi, E., Sirianni, M., Nota, A., et al. 2007, AJ, 133, 44

Searle, L., Sargent, W. L. W., \& Bagnuolo, W. G. 1973, ApJ, 179, 427

Selman, F. J., \& Melnick, J. 2013, A\&A, 552, A94

Selman, F. J., Melnick, J., Bosch, G., \& Terlevich, R. 1999, A\&A, 347, 532

Shapley, A. E., Steidel, C. C., Pettini, M., \& Adelberg, K. L. 2003, ApJ, 588, 65

Skrutskie, M. F., Cutri, R. M., Stiening, R., et al. 2006, AJ, 131, 1163

Smith, R., Slater, R., Fellhauer, M., Goodwin, S., \& Assmann, P. 2011, MNRAS, 416, 383

Stinson, G. S., Dalcanton, J. J., Quinn, T., Kaufmann, T., \& Wadsley, J. 2007, ApJ, 667, 170

Tolstoy, E., Hill, V., \& Tosi, M. 2009, ARA\&A, 47, 371

Tosi, M., Greggio, L., \& Focardi, P. 1989, Ap\&SS, 156, 295

Townsley, L. K., Broos, P. S., Feigelson, E. D., et al. 2006, AJ, 131, 2140

Vanhala, H. A. T., \& Cameron, A. G. W. 1998, ApJ, 508, 291

Walborn, N., \& Blades, J. C. 1997, ApJS, 112, 457

Walborn, N. R. 1973, ApJL, 182, L21

Walborn, N. R., Barbá, R., \& Sewillo, M. 2013, AJ, 145, 98

Walborn, N. R., Barbá, R. H., Brandner, W., et al. 1999, AJ, 117, 225

Walborn, N. R., Maíz Apellániz, J., \& Barbá, R. H. 2002, AJ, 124, 1601

Wang, Q., \& Helfand, D. J. 1991, ApJ, 370, 541

Weigelt, G., \& Baier, G. 1985, A\&A, 150, L18

Zaritsky, D. 1999, AJ, 118, 2824 